\title{
Dual periodicities in the flapping of Saturn's magnetodisk
}

\author{
K. Szego, ${ }^{1}$ Z. Nemeth, ${ }^{1}$ L. Foldy, ${ }^{1}$ S. W. H. Cowley, ${ }^{2}$ and G. Provan ${ }^{2}$ \\ Received 30 January 2013; revised 29 March 2013; accepted 4 May 2013; published 6 June 2013.
}

[1] In this report, we modify the model of Arridge et al. (2011) which was proposed to describe the shape of the magnetodisk of Saturn and its north-south oscillations near the planetary rotation period. The modification is based on the recent results of Provan et al. (2011, 2012) and Andrews et al. (2012), who have shown that the magnetic modulations near the current sheet exhibit dual periodicities, and derived magnetic phase functions for the northern and southern period modulations from the magnetic field data. Using a modified model in which the effects of both modulations are included, specifically related to the modulations of the radial component of the magnetic field, we show that the oscillations can be modeled without the use of an arbitrarily assigned oscillation phase value as employed in the previous work of Szego et al. (2012). In addition, the best fit amplitudes of the two oscillations are found to be nearly constant for a majority of the magnetodisk data analyzed, such that a single model with one fixed parameter fits well to the data for a majority of the passes.

Citation: Szego, K., Z. Nemeth, L. Foldy, S. W. H. Cowley, and G. Provan (2013), Dual periodicities in the flapping of Saturn's magnetodisk, J. Geophys. Res. Space Physics, 118, 2883-2887, doi:10.1002/jgra.50316.

\section{Introduction}

[2] It is well known that Saturn's huge rotating magnetosphere forms a magnetodisk in the equatorial region, in which the fields and particles exhibit rotating periodic modulations near the planetary rotation period in all plasma parameters [e.g., Burch et al., 2009; Andrews et al., 2010]. In the Saturn kilometric radiation (SKR) emissions, two components have been observed since 2007 that have different rotational modulation periods in the northern and southern hemispheres [Gurnett et al., 2009, 2011; Lamy, 2011], with corresponding periods in the field data that briefly coalesced, but did not cross, 1 year after Saturn's vernal equinox in August 2009 [Andrews et al., 2012]. In this brief report, we consider Cassini observations of the oscillating magnetodisk, following the previous analysis by Szego et al. [2012 referred to hereafter as SZ], that were obtained in an interval spanning equinox between spring and autumn 2009. Both magnetic field and SKR data show that the two periods during this interval were coalescing but still distinct, with northern and southern values centered near 10.63 and 10.77 h, respectively. Andrews et al. [2012] also showed that the two field modulations, which are superposed in the equatorial magnetodisk and in the nightside plasma sheet beyond [Provan et al., 2011, 2012], have

\footnotetext{
${ }^{1}$ Institute for Particle and Nuclear Physics, Wigner Research Center for Physics, Budapest, Hungary.

${ }^{2}$ Department of Physics and Astronomy, University of Leicester, Leicester, UK

Corresponding author: K. Szego, Institute for Particle and Nuclear Physics, Wigner Research Center for Physics, Konkoly Thege str. 29-33, Bldg. III, Budapest H-1121, Hungary. (szego.karoly@wigner.mta.hu)

(C)2013. American Geophysical Union. All Rights Reserved 2169-9380/13/10.1002/jgra.50316
}

approximately equal amplitudes during this interval. Here we employ the phase functions for the northern and southern modulations recently derived by Andrews et al. [2012] to reconsider the magnetodisk data discussed by SZ. Further discussion of relevant background may be found in SZ; here we have mentioned only those findings which are new and relevant to the present work.

\section{Analysis and Results}

[3] We focus on the north-south oscillatory motion (flapping) of the magnetodisk current sheet associated with the planetary period modulations. (The coordinates employed are the Saturn-centered SSQ system, in which the $z$ axis is parallel to the rotation and magnetic axes, the solar direction lies in the $z-x$ plane, and $y$ completes the right-handed system pointing from dawn to dusk.) Arridge et al. [2011] proposed a model formula to describe the position of the center of the magnetodisk, employed within models of the magnetic field and plasma sheet, which comprises two terms. The first describes the average location of the magnetodisk as a bowl shape, considered to result from the magnetospheric interaction with the solar wind

$$
z_{\mathrm{CSo}}(\rho)=\left[\rho-r_{H} \tanh \left(\rho / r_{H}\right)\right] \tan \theta_{\text {sun }},
$$

where $\rho$ is the cylindrical radial distance from Saturn's spin and magnetic axis, $r_{H}$ is the hinging distance, and $\theta_{\text {sun }}$ is the latitude of the Sun. The second periodic term added to the first defines the flapping motion for $\rho>\rho_{o}$

$$
\Delta z_{\mathrm{CS}}(\rho)=\left(\rho-\rho_{o}\right) \tan \theta_{\text {tilt }} \cos \left(\Psi_{\mathrm{PS}}(t, \varphi, \rho)\right),
$$

where $\rho_{o}$ is the cylindrical radial distance where the plasma sheet becomes tilted, $\theta_{\text {titt }}$ is the tilt angle of the model plasma 
sheet, and $\Psi_{\mathrm{PS}}(t, \varphi, \rho)$ defines the phase of the plasma/current sheet oscillations near the planetary period, given by

$$
\Psi_{\mathrm{PS}}(t, \varphi, \rho)=\Phi(t)-\varphi-\lambda(\rho)-\psi
$$

[4] In this expression, $\Phi(t)$ is the phase function whose gradient with respect to time $t$ gives the instantaneous period of the oscillations, $\varphi$ is the azimuth around the spin and magnetic axis measured from noon in the sense of planetary rotation (thus related directly to local time) such that the modulations also rotate around the planet in this sense, $\lambda(\rho)$ describes the radial propagation of the oscillations for $\rho>\rho_{o}$ given by $\lambda(\rho)=g\left(\rho-\rho_{o}\right)$ for constant radial phase gradient $g$, and $\psi$ is a constant determined by fits to the data on a pass-by-pass basis. When $\psi=0$ (modulo $360^{\circ}$ ), the function $\Psi_{\mathrm{PS}}(t, \varphi, \rho)$ corresponds to the modulation phase of the radial component of the oscillatory magnetic perturbation field (varying as $\cos \Psi$ ). The connection specifically to $B_{r}$ follows from the definition of the magnetodisk center as the point where the radial field reverses sign, which given Saturn's field polarity is displaced to the south when a positive (outward) radial field is superimposed and to the north for a negative (inward) radial field (see the discussion in Provan et al. [2012]). We also note that the heavy ions accumulate and peak in density close to the magnetodisk center, but not necessary exactly at the center, as shown by Szego et al. [2011] for some passes before May 2008. The difference in time along the Cassini orbit is small, however, less than $15 \mathrm{~min}$.

[5] Since the magnetic perturbations of both northern and southern systems are superimposed in the equatorial region in both the magnetodisk and nightside plasma sheet [Provan et al., 2011, 2012; Andrews et al., 2012], both must contribute to the varying position of the center of the current sheet. Arridge et al. [2011] included only the effect of the southern oscillations that were dominant during southern solstice conditions early in the Cassini mission. In the equinoctial data considered here, however, where the two oscillations are of near-equal amplitude, both should be included. Since the displacement of the sheet center must depend on the total radial perturbation field, which is the linear sum of the northern $(\mathrm{N})$ and southern (S) perturbation fields, we modify equation (2) by replacing the $\cos \left(\Psi_{\mathrm{PS}}(t, \varphi, \rho)\right)$ term by

$$
a_{1} \cos \left(\Phi_{N}(t)-\varphi-\lambda(\rho)\right)+a_{2} \cos \left(\Phi_{S}(t)-\varphi-\lambda(\rho)\right)
$$

where $\Phi_{N}$ and $\Phi_{S}$ are the northern and southern phase functions for the radial fields derived by Andrews et al. [2012], the $a_{1}$ and $a_{2}$ parameters allow variation of the relative amplitudes of the superposed oscillations, and the other terms are as in equation (3). We note, however, that there is now no constant phase terms $\psi$ to be determined from the fit. We also note that in the radial propagation term $\lambda(\rho)$ we employ a phase gradient $g=2.5^{\circ} R_{S}{ }^{-1}$ beyond $\rho_{\mathrm{o}}=12 R_{S}$, according to the results of Provan et al. [2012]. ( $R_{S}$ is Saturn equatorial 1 bar radius equal to $60,268 \mathrm{~km}$.) In the literature [e.g., Arridge et al., 2011], phase delays between $2.5^{\circ}$ and $4.7^{\circ} R_{S}{ }^{-1}$ have been found, equivalent to phase corrections in the range of $20^{\circ}-34^{\circ}$ for wave propagation from $12 R_{S}$ to $20 R_{S}$. This small variation does not affect our fit and could be corrected by a small modification of the amplitude.

[6] As indicated above, we investigate the same data set as $\mathrm{SZ}$, that is ion densities derived from numerical moments
[Thomsen et al., 2010] measured by the Cassini Plasma Spectrometer [Young et al., 2004]. We use measurements in the time range DOY 092-285 2009, spanning Saturn equinox (DOY 223 2009), along nine orbits of the Cassini spacecraft (revolutions 108-119), each of which is about 3 days long and included a Titan flyby. The first test we used on the phase function in equation (4) is how well it reproduces the measured variations of $B_{r}$ for all orbits in question. This test was passed successfully, as expected, so we do not reproduce it here.

[7] One of the main findings in SZ was that the Arridge et al. [2011] model agrees well with the thermal ion density observations, but each pass required different amplitudes $\tan \theta_{\text {tilt }}$ and phase constants $\psi$ to obtain a good fit to the data. As noted above, however, in the modified model using equation (4), there is no freedom concerning the phase, and the only free parameters are the magnitudes of the amplitude factors $a_{1}$ and $a_{2}$. Here we kept the ratio of the northern to southern amplitude fixed at $\left(a_{1} / a_{2}\right)=0.95$, in agreement with the near-equal magnetic field amplitudes found by Andrews et al. [2012], and varied only one amplitude parameter for each pass, corresponding to the factor $a_{1} \tan \theta_{\text {tilt }}$. In Figure 1, as an example, we exhibit the old and new fits for pass T58, to be discussed in more detail below; in Figure 2 two more cases are shown. (We note that the ion and magnetic perturbations occurring near 2009-189 17:04 are due to a Titan encounter and are thus not part of the periodic behavior.) The fit for this and other passes for the amplitude factor $a_{1} \tan \theta_{\text {tilt }}$ is shown in Table 1 . Modest variations in the $\left(a_{1} / a_{2}\right)$ ratio were found not to greatly influence the fit. It is thus notable that for a majority of the passes, an almost fixed value of the amplitude factor $\sim 0.15-0.2$ fits the data well. Thus, the need for varying phase constants and differing amplitudes is largely avoided using the modified model; the phase predicted by equation (4) results in a good fit to all passes, and the amplitude function is generally not strongly varying. This represents a significant improvement over previous results.

[8] We now return to Figure 1 to discuss the data and the fit in more detail. All the horizontal axes show time in DOY 2009. The first panel shows $1 \mathrm{~min}$ averaged magnetic field data in the KRTP spherical polar coordinate system (e $\mathbf{e}_{r}$ is directed radially outward from Saturn along the line connecting Saturn and Cassini, $\mathbf{e}_{\theta}$ is in the co-latitudinal direction, and $\mathbf{e}_{\varphi}$ is in the azimuthal direction and is positive in the prograde direction); the $B_{r}$ component is shown in black, $B_{\theta}$ in medium purple, $B_{\varphi}$ in light green, and the total field strength $B_{\text {tot }}$ in the thick red line. The second panel shows the modulo $360^{\circ} \mathrm{SKR}$ (top) and magnetic field (bottom) phases for the northern (green) and southern (black) modulations, with $0^{\circ}$ to $360^{\circ}$ scales as indicated on the left and right axes. In the plot, the SKR SLS4 longitude system is used [Gurnett et al., 2011], available from the http:// www-pw.physics.uiowa.edu/sls4/ website. The third and fourth panels show the proton and heavy-ion densities (in particles $\mathrm{cm}^{-3}$ ) from the numerical moment calculations. The "good view" part of the data (for which the direction of the ion flow was inside the field of view of the CAPS instrument) are distinguished from other reliable data by color; the lines are green for "good view," and are black for the other reliable density moments. The fifth and sixth panels then illustrate the comparison of the data and the two models for 

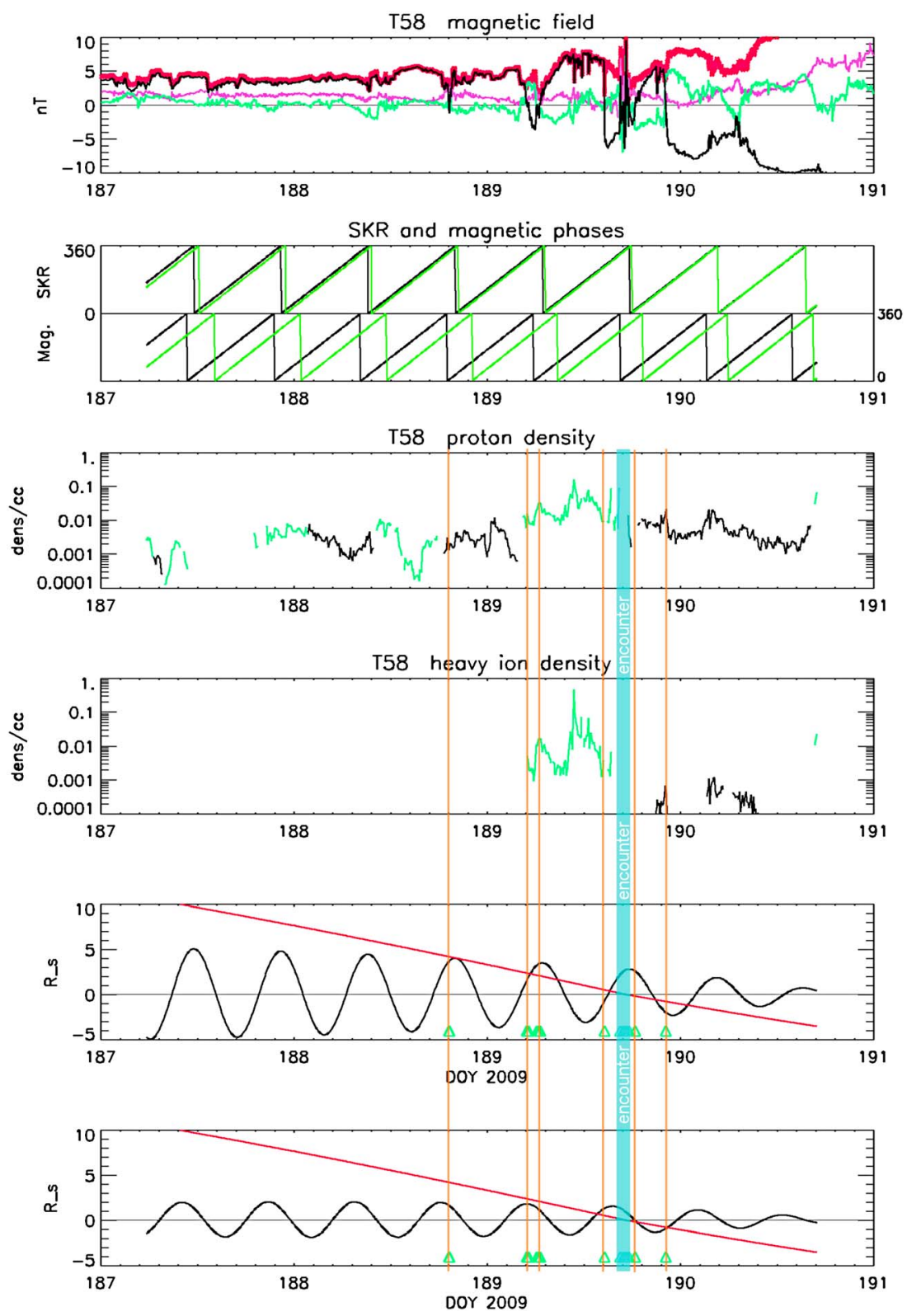

Figure 1. All the horizontal axes are time in DOY 2009. The first panel shows 1 min averaged magnetic field data, where $B_{r}$ is shown in black, $B_{\theta}$ in medium purple, $B_{\varphi}$ in light green, and the total field strength $B_{\text {tot }}$ in thick red line. The second panel shows the modulo $360^{\circ}$ SKR (top) and magnetic field (bottom) phases for the northern (green) and southern (black) modulations, with $0^{\circ}$ to $360^{\circ}$ scales as indicated on the left and right axes. Here the SKR SLS4 longitude system has been used [Gurnett et al., 2011]. The third and fourth panels show the proton and heavy-ion densities (in particles $\mathrm{cm}^{-3}$ ) from the numerical moment calculations. The "good view" part of the data (for which the direction of the ion flow was inside the field of view of the CAPS instrument) is distinguished from other reliable data by color; the lines are green for "good view" and are black for the other reliable density moments. The fifth and sixth panels then illustrate the comparison of the data and the two models for the position of the center of the magnetodisk. The red lines show the displacement (in $R_{S}$ ) of the spacecraft along the planet's spin and magnetic axis from the equatorial plane, positive northward, while the green triangles on the horizontal axes indicate the locations where the radial component is observed to change sign. The orange vertical lines also mark some of these locations. Several green triangles are due to a Titan encounter and are thus not part of the periodic behavior; we have covered those with a light blue vertical bar. The black lines show the fits for the position of the model current sheet $z_{\mathrm{CS}}$, where the fifth panel shows the previous fit from SZ while the sixth panel shows the new fit using equation (4). 

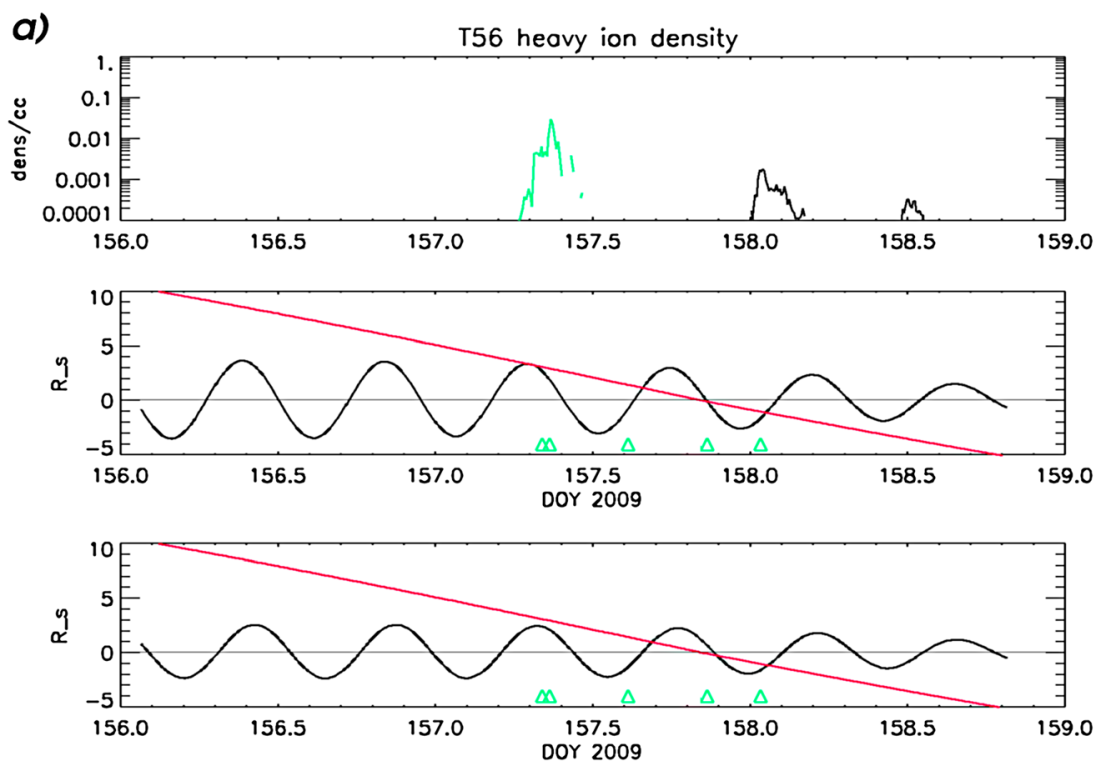

b)
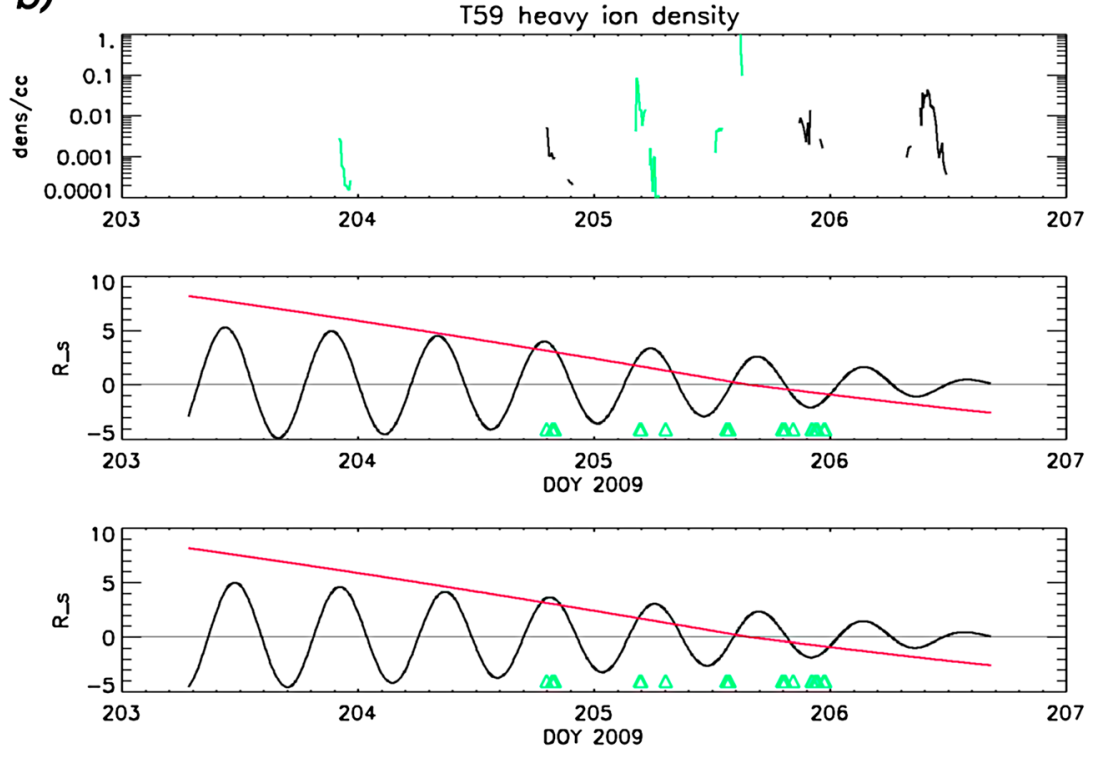

Figure 2. Data equivalent to the fourth to sixth panels of Figure 1 are shown for two more flybys. The format is the same as in Figure 1; except that green triangles for the Titan closest approach are not shown.

the position of the center of the magnetodisk. The red lines show the displacement (in $R_{S}$ ) of the spacecraft along the planet's spin and magnetic axis from the equatorial plane, positive northward, while the green triangles on the horizontal axes indicate the locations where the radial component is observed to change sign. The orange vertical lines also mark some of these locations. Several green triangles are due to a Titan encounter and are thus not part of the periodic behavior; we have covered these with a light blue vertical bar. The black lines show the fits for the position of the model current sheet $z_{\mathrm{CS}}$, where the fifth panel shows the previous fit from SZ while the sixth panel shows the new fit using equation (4). We note that the amplitude of the fitted black line varies significantly over the interval due to variation of the $\left(\rho-\rho_{\mathrm{o}}\right)$ factor in equation (2). In Figure 2, the last three plots of Figure 1 are shown for two more passes. Both in
Figures $2 \mathrm{a}$ and $2 \mathrm{~b}$, the green triangles are not shown for closest approach interval.

[9] The drivers for the fit were (a) to relate the observed zero points of $B_{r}$ with the locations where the spacecraft

Table 1. The Parameters of the Fit

\begin{tabular}{lc}
\hline Passes & Fitted Amplitude Value for $a_{1} \tan \theta_{\text {tilt }}$ \\
\hline T52 & 0.07 \\
T53 & 0.25 \\
T55 & 0.30 \\
T56 & 0.20 \\
T57 & 0.20 \\
T58 & 0.15 \\
T59 & 0.15 \\
T61 & 0.15 \\
T62 & 0.15 \\
\hline
\end{tabular}


crosses the model center plane and (b) to minimize the distance between the model center plane and the locations of peak ion densities. The main strength of the new fit, however, is that the same phase function works for all the passes we have investigated, together with almost constant amplitudes; the latter with the exception of passes T52 and T55. This clearly represents a significant improvement in the model. We note, however, that the densities in the magnetodisk are observed to fluctuate from pass to pass; this dynamic behavior possibly being reflected in the small changes in best fit amplitude. It is not possible to characterize quantitatively the fit. Whereas condition (a) could easily be quantified, condition (b) is mathematically not strict enough, since we see on the data plots that peak ion densities are not necessarily at the same locations for the two ion components, and the locations of the peak ion densities and the zero points of $B_{r}$ can also be shifted relative to each other. It should not be forgotten that what we fit is the average magnetodisk location, while the real magnetodisk very likely is more structured. In SZ, we emphasized that the fit of $z_{\mathrm{CS}}$ (the vertical position of the magnetodisk above the equator) is "visual," because we do not have really strict mathematical conditions to optimize. The same is true here, such that all possible "quantitative criteria" would be just as subjective as our "by the eye" method. Furthermore, we do not claim that the fit is better in the sense that it provides significantly closer fits for individual curves. But it is better in the sense that now we do not need free phase parameters. One arbitrary fit parameter has been removed, so we are fitting with only the amplitude free, and that turns out mostly to be similar from pass to pass. This means that we have predictive power. An equal fit with less fit parameters and more predictive power represents significant progress in our opinion.

[10] In the model of Jia and Kivelson [2012], a certain asymmetry was observed in the "flapping" motion, with the disk being more frequently above the geometric equator than below, though for a different time period. This is not the case in our fit despite their model geometry being more similar to the 2009 situation investigated here, with the angle subtended by the solar wind and the equatorial plane being zero in their model. However, they used an asymmetric ionospheric current system as an input for their numerical model, with stronger currents in the south than in the north, which is not applicable to our equinox conditions where the two current systems are known to be closely comparable [Andrews et al., 2012]. This may be the cause of the north-south asymmetry in their results which is not reproduced here.

[11] We note that the modified model also has important consequences for Titan's interaction with the magnetospheric plasma. Using the new model, the location of the magnetodisk relative to Titan can be predicted for each flyby, making it easier to understand the relative position of Titan and the disk and how this relative position changes during the flyby. This will help to improve the predictive power of the models of Titan's magnetic environment.

\section{Conclusions}

[12] In summary, we report here a new model for the northsouth motion of the magnetodisk in the nightside during equinoctial conditions. The basis of the model is the observed dual periodicity of the modulation of the magnetic field near the equatorial plane [Provan et al., 2011, 2012], and the numerical determination of those phases by Andrews et al. [2012]. The strength of the new model is that the phase of the motion can be predicted from the phase of the radial magnetic field modulations alone, without using any additional free phase fitting parameter. The model has been verified using CAPS numerical moments, though only on a limited data set of nine passes so far. The new model provides a better fit to the data, with less free parameters. We believe that this is an important step forward.

[13] Acknowledgments. Work at Leicester was supported by STFC grant ST/H002480/1.

[14] Masaki Fujimoto thanks the reviewers for their assistance in evaluating this paper.

\section{References}

Andrews, D. J., S. W. H. Cowley, M. K. Dougherty, and G. Provan (2010), Magnetic field oscillations near the planetary period in Saturn's equatorial magnetosphere: Variation of amplitude and phase with radial distance and local time, J. Geophys. Res., 115, A04212, doi:10.1029/2007JA014729.

Andrews, D. J., S. W. H. Cowley, M. K. Dougherty, L. Lamy, G. Provan, and D. J. Southwood (2012), Planetary period oscillations in Saturn's magnetosphere: Evolution of magnetic oscillation properties from southern summer to postequinox, J. Geophys. Res., 117, A04224, doi:10.1029/2011JA017444.

Arridge, C. S., et al. (2011), Periodic motion of Saturn's nightside plasma sheet, J. Geophys. Res., 116, A11205, doi:10.1029/2011JA016827.

Burch, J. L., A. D. DeJong, J. Goldstein, and D. T. Young (2009), Periodicity in Saturn's magnetosphere: Plasma cam, Geophys. Res. Lett., 36, L14203, doi:10.1029/2009GL039043.

Gurnett, D. A., A. Lecacheux, W. S. Kurth, A. M. Persoon, J. B. Groene, L. Lamy, P. Zarka, and J. F. Carbary (2009), Discovery of a north-south asymmetry in Saturn's radio rotation period, Geophys. Res. Lett., 36, L16102, doi:10.1029/2009GL039621.

Gurnett, D. A., J. B. Groene, T. F. Averkamp, W. S. Kurth, S.-. Y. Ye, and G. Fischer (2011), The SLS4 longitude system based on a tracking filter analysis of the rotational modulation of Saturn kilometric radiation, in Planetary Radio Emissions VII, edited by H. O. Rucker, W. S. Kurth, P. Louarn, and G. Fischer, pp. 51-64, Austrian Acad. Sci. Press, Vienna.

Jia, X., and M. G. Kivelson (2012), Driving Saturn's magnetospheric periodicities from the upper atmosphere/ionosphere: Magnetotail response to dual sources, J. Geophys. Res., 117, A11219, doi:10.1029/ 2012JA018183.

Lamy, L. (2011), Variability of southern and northern SKR periodicities, in Planetary Radio Emissions VII, edited by H. O. Rucker, W. S. Kurth, P. Louarn, and G. Fischer, pp. 39-50, doi:10.1553/PRE7s39, Austrian Acad. Sci. Press, Vienna.

Provan, G., D. J. Andrews, B. Cecconi, S. W. H. Cowley, M. K. Dougherty, L. Lamy, and P. Zarka (2011), Magnetospheric period magnetic field oscillations at Saturn: Equatorial phase "jitter" produced by superposition of southern- and northern-period oscillations, J. Geophys. Res., 116, A04225, doi:10.1029/2010JA016213.

Provan, G., D. J. Andrews, C. S. Arridge, A. J. Coates, S. W. H. Cowley, G. Cox, M. K. Dougherty, and C. M. Jackman (2012), Dual periodicities in planetary-period magnetic field oscillations in Saturn's tail, J. Geophys. Res., 117, A01209, doi:10.1029/2011JA017104.

Szego, K., Z. Nemeth, G. Erdos, L. Foldy, M. Thomsen, and D. Delapp (2011), The plasma environment of Titan: The magnetodisk of Saturn near the encounters as derived from ion densities measured by the Cassini/CAPS plasma spectrometer, J. Geophys. Res., 116, A10219, doi:10.1029/2011JA016629.

Szego, K., Z. Nemeth, G. Erdos, L. Foldy, Z. Bebesi, M. Thomsen, and D. Delapp (2012), Location of the magnetodisk in the nightside outer magnetosphere of Saturn near equinox based on ion densities, J. Geophys. Res., 117, A09225, doi:10.1029/2012JA017817.

Thomsen, M. F., D. B. Reisenfeld, D. M. Delapp, R. L. Tokar, D. T. Young, F. J. Crary, E. C. Sittler, M. A. McGraw, and J. D. Williams (2010), Survey of ion plasma parameters in Saturn's magnetosphere, J. Geophys. Res., 115, A10220, doi:10.1029/2010JA015267

Young, D. T. et al. (2004), Cassini plasma spectrometer investigation, Space Sci. Rev., 114, 1-112, doi:10.1007/s11214-004-1406-4. 